\title{
Assessment of the subclinical myocardial dysfunction with preserved left ventricle ejection fraction in type 2 diabetes mellitus patients with global myocardial work
}

\section{Tao Wang}

Changzhou No.2 People's hospital

Jun Huang (D 305669112@qq.com )

Changzhou No.2 people's hospital https://orcid.org/0000-0002-4680-5344

Zi-ning Yan

Changzhou No.2 People's hospital

Li Fan

Changzhou No.2 People's Hospital

\section{Research Article}

Keywords: two-dimensional echocardiography, type 2 diabetes mellitus, myocardial work, myocardial layer-specific strain

Posted Date: February 11th, 2021

DOI: https://doi.org/10.21203/rs.3.rs-210151/v1

License: (1) This work is licensed under a Creative Commons Attribution 4.0 International License. Read Full License 


\section{Abstract}

To assess the subclinical left ventricle(LV) myocardial dysfunction in preserved left ventricle ejection fraction(LVEF) in type 2 diabetes mellitus(T2DM) patients by global myocardial work(MW). 60 untreated T2DM patients and 60 normal controls were enrolled for this study. Apical 4-, 3- and 2-chamber views were acquired by two-dimensional echocardiography. Peak systolic myocardial layer-specific global longitudinal strain(GLS), peak strain dispersion(PSD), global myocardial work index(GWI), global constructive work(GCW), global wasted work(GWW), and myocardial work efficiency(GWE) were generated by speckle-tracking echocardiography(STE). The values of GLS in T2DM patients were significantly lower than normal controls $(p<0.05)$. GWW in T2DM patients was significantly larger than normal controls, while GWE was significantly lower $(p<0.05)$. In T2DM patients, fasting plasma glucose positively correlated with GWW, while negatively correlated with GWE, respectively. ROC analysis showed GWE was superior to other values with a highest AUC of 0.688. Logistic regression analysis indicated that GLSEndo (OR $=1.196,95 \% \mathrm{Cl}: 1.012-1.414, \mathrm{p}=0.036)$, and GWE (OR $=0.585,95 \% \mathrm{Cl}: 0.414-0.825, \mathrm{p}=$ 0.002) were independent risk factors associated with LV function in T2DM patients. From the research, the subclinical LV myocardial dysfunction was detected by GLS and global MW in T2DM patients. Global MW is a sensitive predictor to demonstrate the subclinical LV myocardial dysfunction in T2DM patients.

\section{Introduction}

Type 2 diabetes mellitus (T2DM) is increasing worldwide in recent years. The development of coronary artery disease (CAD) induced by T2DM has been also increased. In the past few years, cardiovascular disease becomes the major cause of morbidity and mortality for T2DM patients[1]. The pathological changes of diabetic induced cardiomyopathy mainly contain cardiomyocyte apoptosis, myocardial fibrosis and necrosis, at last, lead to cardiac systolic and diastolic dysfunction and heart failure[2; 3$]$. Therefore, in order to protect the cardiac function, early detection and intervention the function of left ventricle (LV) is essential to the prevention and management of diabetic cardiomyopathy.

There were a lot of techniques can assess the cardiac function, such as cardiovascular magnetic resonance T1 mapping[4], Tc-99m MIBI gated single-photon emissione-computed tomography (SPECT) myocardial perfusion imaging[5], tissue Doppler strain echocardiography[6], and two-dimensional speckle tracking echocardiography (2D-STE) [7; 8]. However, these techniques are all have their limitations. Like angle-dependence in tissue Doppler strain echocardiography, long-time examination in cardiac MRI and the radioactivity of the cardiac radionuclide imaging. 2D-STE has a lower signal-to-noise ratio, weak acoustic windows, and through-plane motion artifacts[9; 10]. However, 2D-STE has become the leading reliable diagnostic techniques for assessment of the cardiac functions because of its noninvasiveness, convenience, and repeatability. In addition, our group previously reported that LV Iongitudinal myocardial function detected by longitudinal strain and rotation in T2DM patients with preserved LV ejection fraction (LVEF) was impaired when compared with healthy subjects[11]. However, global myocardial work (MW) is a new parameter for 2D-STE that takes into account deformation as well as afterload through 
interpretation of strain in relation to dynamic non-invasive LV pressure, potentially offering incremental value to myocardial function assessment.

The aim of the research was to explore the incremental value of $\mathrm{MW}$ in the detection the subclinical LV myocardial dysfunction of T2DM patients with preserved LV systolic function.

\section{Subject And Methods}

\section{Study population}

60 untreated T2DM patients and 60 normal controls of similar age and gender were enrolled for this study were recruited from the hospital. The inclusion criteria for T2DM patients were clinically confirmed subjects in accordance with the World Health Organization criteria[12] without any history of heart disease (congenital heart disease, coronary artery disease, arterial hypertension, myocardial infarction, cardiomyopathy, valvular disease, atrial fibrillation, thyroid disease, neoplastic disease, or kidney failure). All the T2DM patients had a LVEF > 55\%. The normal controls were recruited from the physical examination or surgery in the hospital. In the normal controls, all of the physical and laboratory examination tests about cardiac function, the electrocardiogram (ECG), and the echocardiography were showed normal.

\section{Anthropometric and biochemistry}

The sex, age, height, body weight, and blood pressure of all subjects were recorded at the time of the echocardiography examination. Fasting plasma glucose, two-hour postprandial blood sugar and glycated hemoglobin (HBA1c) were measured when the patients in hospital.

\section{Conventional 2D Doppler echocardiography}

Conventional 2D transthoracic Doppler echocardiography by Vivid E9 equipped with an M5S 3.5-5 MHz transducer (GE Vingmed Ultrasound, Horten, Norway) were performed to these patients by an experienced cardiologist. All of the patients were connected ECG leads. Apical 4-chamber, 2-chamber, and long-axis views of three consecutive cycles with standard high frame rate $\left(>60 \mathrm{~s}^{-1}\right)$ were stored for offline analysis.

Echocardiographic parameters of left atrial diameter (LAd), interventricular septum thickness (IVSd), LV posterior wall thickness (LVPWd) and LV diameter (LVDd) in end-diastole period, the peak early (E) and late (A) diastolic mitral annular velocities, LV end-diastole volume (LVEDV), LV end-systole volume (LVESV) and LVEF (modified biplane Simpson's method), The peak early (e') and late (a') diastolic annular velocities (obtained by averaging the values at the septum and lateral wall by tissue Doppler imaging (TDI).) were measured during the examination (Figure 1).

\section{Two-Dimensional STE}


Layer specific longitudinal strain (epimyocardial, middle myocardial and endomyocardial), peak strain dispersion (PSD), MW were measured by the software of EchoPAC (EchoPAC Version: 203, GE Vingmed Ultrasound, Norway).

First, identify the aortic valve closure time from the event timing of aortic valve spectrum. Then using the APLAX, A4C and A2C for analysis the apical long-axis, four-chamber and two-chamber views. The LV myocardial was divided into 18 segments, and layer specific global longitudinal strain (GLS) of epimyocardial, middle myocardial and endomyocardial were automatically measured by the software (Figure 2A).

MW and related indices were calculated using a combination of echocardiographic strain data and a noninvasively estimated LV pressure curve. Input the blood pressure into the software, and click the keyboard "Advanced", then MW and related indices were calculated using a combination of echocardiographic strain data and a noninvasively estimated LV pressure curve[13]. (1) GWI: global myocardial work index, (2) GCW: global constructive work. (3) GWW: global wasted work. (4) GWE: myocardial work efficiency, GWE $=\mathrm{GCW} /(\mathrm{GCW} \mathbb{G W W})$.

\section{Statistical analysis}

All data analyses were performed using SPSS 25.0 software (SPSS, Chicago, IL, USA). Data were presented as the mean \pm standard deviation (SD). $p$-value $<0.05$ was considered statistically significant in all tests. Shapiro-Wilk's test or Kolmogorov-Smirnov's test was used to detect the normality of all values. Differences between T2DM patients and normal controls were compared with an independent Student's t-test for the data distribution was normal. For variables with a non-normal distribution, the nonparametric Mann-Whitney test was used. We defined layer specific strains, PSD, MW values in normal controls as the normal state, and considered the values of T2DM patients as abnormal. These values in T2DM patients were determined from receiver operating characteristic (ROC) curve analysis. Yoden's index was selected for the cut-off point which can give the best composite of specificity and sensitivity. Correlations among LVEF, fasting plasma glucose, $\mathrm{HbA1c}$ and layer specific strains, PSD, MW values were tested using Pearson or Spearman correlation tests as appropriate. Logistic regression analysis was performed to assess independent correlates of LV function of T2DM patients. Odds ratio (OR) with $95 \%$ $\mathrm{Cl}$ was estimated.

\section{Reproducibility and repeatability}

Intraobserver and interobserver variability for layer specific GLS, PSD, MW values were determined by repeating measurements in random selected 25 patients among all enrolled patients.

\section{Results}

Patients Characteristics 
120 patients satisfied the baseline inclusion criteria. 19 patients were excluded from strain and MW analysis because of inadequate image quality $(n=12)$, tachycardia $(n=5)$ and irregular heartbeat $(n=2)$. A total of 111 patients were therefore evaluated in the study and were initially divided into two groups, normal controls ( $n=53$, mean age, $49.96 \pm 13.17$ years, 28 men) and T2DM patients $(n=48$, mean age, $53.79 \pm 11.24$ years, men).

\section{Patients characteristics data and conventional echocardiographic parameters in normal controls compared with T2DM patients (Table 1).}

The values of BSA, SBP, fasting plasma glucose and $\mathrm{HbA} 1 \mathrm{c}$ were significantly larger in T2DM patients compared with normal controls $(p<0.05)$. There were no significant differences in age, heart rate, sex and DBP between normal controls and T2DM patients.

The values of LAd, IVSd, a' and E/e' in T2DM patients were significantly larger than normal controls $(p<0.05)$, however, LVEF and e' in T2DM patients were significantly lower than normal controls $(p<0.05)$. There were no significant differences in LVPWd, LVDd, LVEDV, LVESV, E and A between normal controls and T2DM patients $(\mathrm{p}>0.05)$.

\section{GLS of different myocardial layers and global MW between normal controls and T2DM patients (Table 2, Figure 3).}

The values of LV GLS (epimyocardial, middle-layer myocardial and endomyocardial) in T2DM patients were significantly lower than normal controls $(p<0.05)$. The value of PSD in T2DM patients was significantly larger than normal controls $(p<0.05)$. The value of GWW was significantly larger in T2DM patients compared with normal controls, however, the value of GWE was significantly lower in T2DM patients compared with normal controls $(p<0.05)$. There were no significant differences in GWI and GWE between normal controls and T2DM patients( $p>0.05)$, however, the values were a litter lower in T2DM patients when compared with normal controls.

\section{Receiver operating characteristic curve analysis (Table3, Figure 4)}

GWE was superior to other values with an AUC of 0.688 . According to the receiver operating characteristic curve analysis, the optimal cutoff value for GWE for the detection of patients diagnosed was $94 \%$, with sensitivity of $25 \%$, specificity of $100 \%$, and the Youden index was 0.25 .

\section{Correlation tests among LVEF, fasting plasma glucose, HbA1c and LV systolic functions (Figure 5, 6 and 7).}

In total population, LVEF negatively correlated with GLSEpi, GLSMid, and GLSEndo. Fasting plasma glucose positively correlated with GLSEpi, GLSMid, GLSEndo and GWW, while negatively correlated with GWE. HbA1c positively correlated with GLSMid, GLSEndo and GWW, while negatively correlated with GWE. In normal controls, HbA1c positively correlated with GWI, GCW and GWE, while negatively correlated 
with GWW. In T2DM patients, fasting plasma glucose positively correlated with GWW, while negatively correlated with GWE, respectively.

\section{Risk factors for LV function of T2DM patients (Table 4).}

Logistic regression analysis was applied, where the variables were selected because they were associated with LV function of T2DM patients with a p value < 0.05 (LVEF, GLSEpi, GLSMid, GLSEndo, PSD, GWW, GWE). It indicated that GLSEndo (OR=1.196, 95\% Cl: 1.012-1.414, $p=0.036)$, and GWE (OR=0.585, 95\% Cl: 0.414-0.825, $p=0.002)$, were independent risk factors associated with LV function of T2DM patients.

\section{Intra- and Interobserver Variability (Table 5)}

Intraclass correlation coefficient (ICC) of measurement by intra- and interobserver variabilities were calculated. All GLS of different layers and global MW parameters showed excellent intra- and interobserver correlation with ICC values $>0.85$.

\section{Discussion}

The main finding of the study was: the subclinical LV myocardial dysfunction was detected in T2DM patients with preserved LV systolic function. Global MW was a sensitive predictor for detecting the subclinical LV myocardial dysfunction in T2DM patients. Fasting plasma glucose maybe a predict factor to cardiac MW.

The cardiac impaired by T2DM contained microvascular impairment, metabolic disturbance, subcellular component abnormalities, cardiac autonomic dysfunction, and a maladaptive immune response. Eventually, functional and structural changes in the myocardium without coronary artery disease were caused by T2DM, the disorder is called diabetic cardiomyopathy[14]. Diabetic cardiomyopathy may first induce diastolic dysfunction, and then systolic dysfunction. Lastly, the clinical heart failure maybe occurred. With the development of image examination, detection the cardiac function becomes ever more and more easy. Echocardiography for measuring the MW was considered the newest tool for the assessment of LV systolic dysfunction. Billig S[15], et al evaluated left and right ventricular structure, function and myocardial work by transesophageal echocardiography (TEE) in swine, and found that myocardial contractility and mechanics could be reliably evaluated with the non-invasive GWI derived from echocardiography without additional invasive measures. Galli E[16], et al provided the reference values for GWI, GCW, GWW and GWE in a group of healthy volunteers accounting for age and gender, and found that the assessment of MW is feasible in normal subjects. The presented referral ranges of GWI, GCW, GWW and GWE were not affected by age. According to the previous studies, MW have been reported in the coronary heart disease[17], hypertension, cardiac resynchronization therapy(CRT), percutaneous coronary intervention (PCI)[18], aortic stenosis[19], transcatheter aortic valve replacement (TAVR)[20], dilated cardiomyopathy[21], hypertrophic cardiomyopathy[22], chronic kidney disease[23], cardiac amyloidosis[24] and so on. However, there was little research about the LV systolic function in T2DM patients with MW. 
Liu JH[25], et al provided prognostic value in T2DM patients by GLS and found that in T2DM patients without any history of cardiovascular disease, impaired GLS was associated with cardiovascular events. Our research was according with this research, and we found that layer-specific LV GLS was decreased in T2DM patients when compared with normal controls. MW analysis had shown that the value of GWW was significantly larger in T2DM patients compared with normal controls and the value of GWE was significantly lower in T2DM patients compared with normal controls. Although there were no significant differences in GWI and GWE between normal controls and T2DM patients, the values were a litter lower in T2DM patients when compared with normal controls. The results may conclude that the subclinical LV myocardial dysfunction was detected in T2DM patients although the LV systolic function was normal. We considered the results were related to the pathological changes of LV myocardium in T2DM. In T2DM, persistent hyperglycemia causes the molecular and metabolic changes in cardiomyocytes and damages the coronary microvasculature. Hypoxia of cardiomyocytes and ischemia results myocardial hypertrophy, perivascular and fibrosis, LV stiffness, and systolic and diastole dysfunction in T2DM[14]. A normal myocardial fiber consists of longitudinal and circumferential myocardial fibers. Almost $70 \%$ of the myocardial fiber is longitudinal and $30 \%$ is circumferential. If there was cardiomyocyte apoptosis, myocardial fibrosis and necrosis in T2DM, the sequence of myocardium may change, and eventually lead to the damage of myocardial systolic function, also the reduced PSD could prove the dysfunction.

In total population, LVEF negatively correlated with GLSEpi, GLSMid, and GLSEndo. Fasting plasma glucose positively correlated with GLSEpi, GLSMid, GLSEndo and GWW, while negatively correlated with GWE. HbA1c positively correlated with GLSMid, GLSEndo and GWW, while negatively correlated with GWE. In normal controls, HbA1c positively correlated with GWI, GCW and GWE, while negatively correlated with GWW. In T2DM patients, fasting plasma glucose positively correlated with GWW, while negatively correlated with GWE, respectively.

In this study, we evaluated the risk factors of LV function in T2DM patients. It indicated that GLSEndo $(\mathrm{OR}=1.196,95 \% \mathrm{Cl}: 1.012-1.414, \mathrm{P}=0.036)$, and GWE (OR $=0.585,95 \% \mathrm{Cl}: 0.414-0.825, \mathrm{P}=.002)$, were independent risk factors associated with LV function of T2DM patients. Thus, taking into account for GLSEndo and GWE status might further improve LV function of T2DM patients.

\section{Conclusion}

From the research, we found that the subclinical LV myocardial dysfunction is detected in T2DM patients with preserved LV systolic function. Global MW is a sensitive predictor to demonstrate the subclinical LV systolic dysfunction in T2DM patients with preserved LV systolic function. Different levels of fasting plasma glucose maybe a predict factor for MW in T2DM patients.

\section{Declarations}

\section{Conflict of interest}


The authors declare that they have no conflict of interest.

\section{Ethical statement}

The study conducted according to the Principles of the Declaration of Helsinki and was approved by the human research and ethics committee of the affiliated Changzhou No.2 people's hospital with Nanjing Medical University. All of the patients had completed the informed consent forms.

\section{Funding Sources}

This study was funded by Changzhou Science and Technique Program (Grant No.: CJ20190098), Young Talent Development Plan of Changzhou Health Commission (No.: CZQM2020061) and Talent Development Plan of Changzhou No.2 People's hospital (No.: YJRC202031).

\section{Acknowledgements}

We would like to thanks the department of Radiology and Echocardiography, the Affiliated Changzhou No.2 People's Hospital with Nanjing Medical University.

\section{References}

1 Low Wang CC, Hess CN, Hiatt WR, Goldfine AB (2016) Clinical Update: Cardiovascular Disease in Diabetes Mellitus: Atherosclerotic Cardiovascular Disease and Heart Failure in Type 2 Diabetes Mellitus Mechanisms, Management, and Clinical Considerations. Circulation 133:2459-2502

2 Bugger H, Abel ED (2014) Molecular mechanisms of diabetic cardiomyopathy. Diabetologia 57:660-671

3 Aneja A, Tang WH, Bansilal S, Garcia MJ, Farkouh ME (2008) Diabetic cardiomyopathy: insights into pathogenesis, diagnostic challenges, and therapeutic options. Am J Med 121:748-757

4 Cao Y, Zeng W, Cui Y et al (2018) Increased myocardial extracellular volume assessed by cardiovascular magnetic resonance T1 mapping and its determinants in type 2 diabetes mellitus patients with normal myocardial systolic strain. Cardiovasc Diabetol 17:7

5 Deepti S, Roy A, Patel CD et al (2018) Assessment of asymptomatic ischemic heart disease using stress myocardial perfusion imaging in patients with type 2 diabetes mellitus. Indian Heart J 70 Suppl 3:S157S160

6 Galderisi M, de Simone G, Innelli P et al (2007) Impaired inotropic response in type 2 diabetes mellitus: a strain rate imaging study. Am J Hypertens 20:548-555

7 Ernande L, Bergerot C, Girerd N et al (2014) Longitudinal myocardial strain alteration is associated with left ventricular remodeling in asymptomatic patients with type 2 diabetes mellitus. J Am Soc Echocardiogr 27:479-488 
$8 \mathrm{Ng}$ AC, Delgado V, Bertini M et al (2010) Myocardial steatosis and biventricular strain and strain rate imaging in patients with type 2 diabetes mellitus. Circulation 122:2538-2544

9 Pedrizzetti G, Claus P, Kilner PJ, Nagel E (2016) Principles of cardiovascular magnetic resonance feature tracking and echocardiographic speckle tracking for informed clinical use. J Cardiovasc Magn Reson 18:51

10 Scatteia A, Baritussio A, Bucciarelli-Ducci C (2017) Strain imaging using cardiac magnetic resonance. Heart Fail Rev 22:465-476

11 Huang J, Hu HL, Yan ZN et al (2019) Peak systolic longitudinal rotation: a new tool for detecting left ventricular systolic function in patients with type 2 diabetes mellitus by two-dimensional speckle tracking echocardiography. BMC Cardiovasc Disord 19:137

12 Alberti KG, Zimmet PZ (1998) Definition, diagnosis and classification of diabetes mellitus and its complications. Part 1: diagnosis and classification of diabetes mellitus provisional report of a WHO consultation. Diabet Med 15:539-553

13 Chan J, Edwards NFA, Khandheria BK et al (2019) A new approach to assess myocardial work by noninvasive left ventricular pressure-strain relations in hypertension and dilated cardiomyopathy. Eur Heart $\mathrm{J}$ Cardiovasc Imaging 20:31-39

14 Lee WS, Kim J (2017) Diabetic cardiomyopathy: where we are and where we are going. Korean J Intern Med 32:404-421

15 Billig S, Zayat R, Ebeling A et al (2020) Transesophageal echocardiography in swine: evaluation of left and right ventricular structure, function and myocardial work. Int J Cardiovasc Imaging. 10.1007/s10554020-02053-7

16 Galli E, John-Matthwes B, Rousseau C, Schnell F, Leclercq C, Donal E (2019) Echocardiographic reference ranges for myocardial work in healthy subjects: A preliminary study. Echocardiography 36:1814-1824

17 Edwards NFA, Scalia GM, Shiino K et al (2019) Global Myocardial Work Is Superior to Global Longitudinal Strain to Predict Significant Coronary Artery Disease in Patients With Normal Left Ventricular Function and Wall Motion. J Am Soc Echocardiogr 32:947-957

18 Meimoun P, Abdani S, Stracchi V et al (2020) Usefulness of Noninvasive Myocardial Work to Predict Left Ventricular Recovery and Acute Complications after Acute Anterior Myocardial Infarction Treated by Percutaneous Coronary Intervention. J Am Soc Echocardiogr 33:1180-1190

19 Fortuni F, Butcher SC, van der Kley F et al (2020) Left ventricular myocardial work in patients with severe aortic stenosis. J Am Soc Echocardiogr. 10.1016/j.echo.2020.10.014 
20 Jain R, Bajwa T, Roemer S et al (2020) Myocardial work assessment in severe aortic stenosis undergoing transcatheter aortic valve replacement. Eur Heart J Cardiovasc Imaging.

10.1093/ehjci/jeaa257

21 Cui C, Liu L, Li Y et al (2020) Left Ventricular Pressure-Strain Loop-Based Quantitative Examination of the Global and Regional Myocardial Work of Patients with Dilated Cardiomyopathy. Ultrasound Med Biol 46:2834-2845

22 Hiemstra YL, van der Bijl P, El Mahdiui M, Bax JJ, Delgado V, Marsan NA (2020) Myocardial Work in Nonobstructive Hypertrophic Cardiomyopathy: Implications for Outcome. J Am Soc Echocardiogr 33:1201-1208

23 Ke QQ, Xu HB, Bai J, Xiong L, Li MM (2020) Evaluation of global and regional left ventricular myocardial work by echocardiography in patients with chronic kidney disease. Echocardiography. 10.1111/echo.14864

24 Clemmensen TS, Eiskjaer H, Ladefoged B et al (2020) Prognostic implications of left ventricular myocardial work indices in cardiac amyloidosis. Eur Heart J Cardiovasc Imaging. 10.1093/ehjci/jeaa097

25 Liu JH, Chen Y, Yuen M et al (2016) Incremental prognostic value of global longitudinal strain in patients with type 2 diabetes mellitus. Cardiovasc Diabetol 15:22

\section{Tables}

Tables 1-5 not available with this version

\section{Figures}

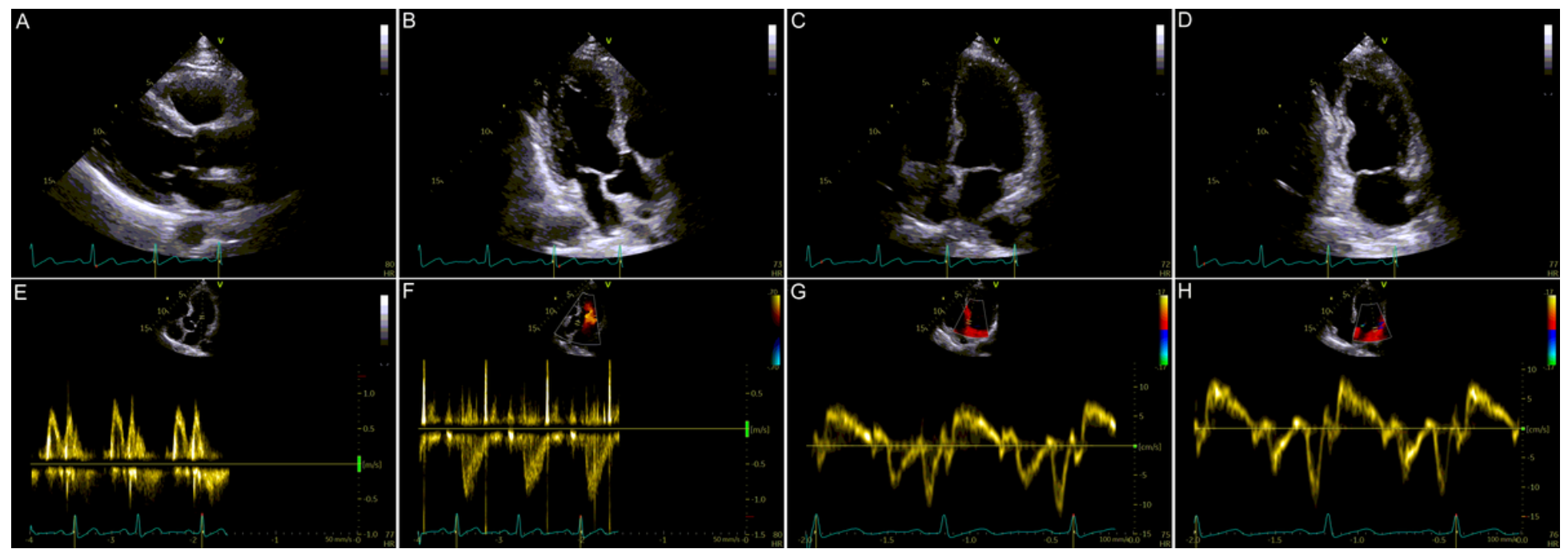

Figure 1 
A: parasternal long-axis view. B: apical long-axis view. C: apical 4-chamber view. D: apical 2-chamber view. E: mitral valve flow spectrum measured by pulsed wave doppler. F: aortic valve flow spectrum measured by pulsed wave doppler G: peak early (e') and late (a') diastolic annular velocities at the septum wall by TDI. H: peak early (e') and late (a') diastolic annular velocities at the lateral wall by TDI.
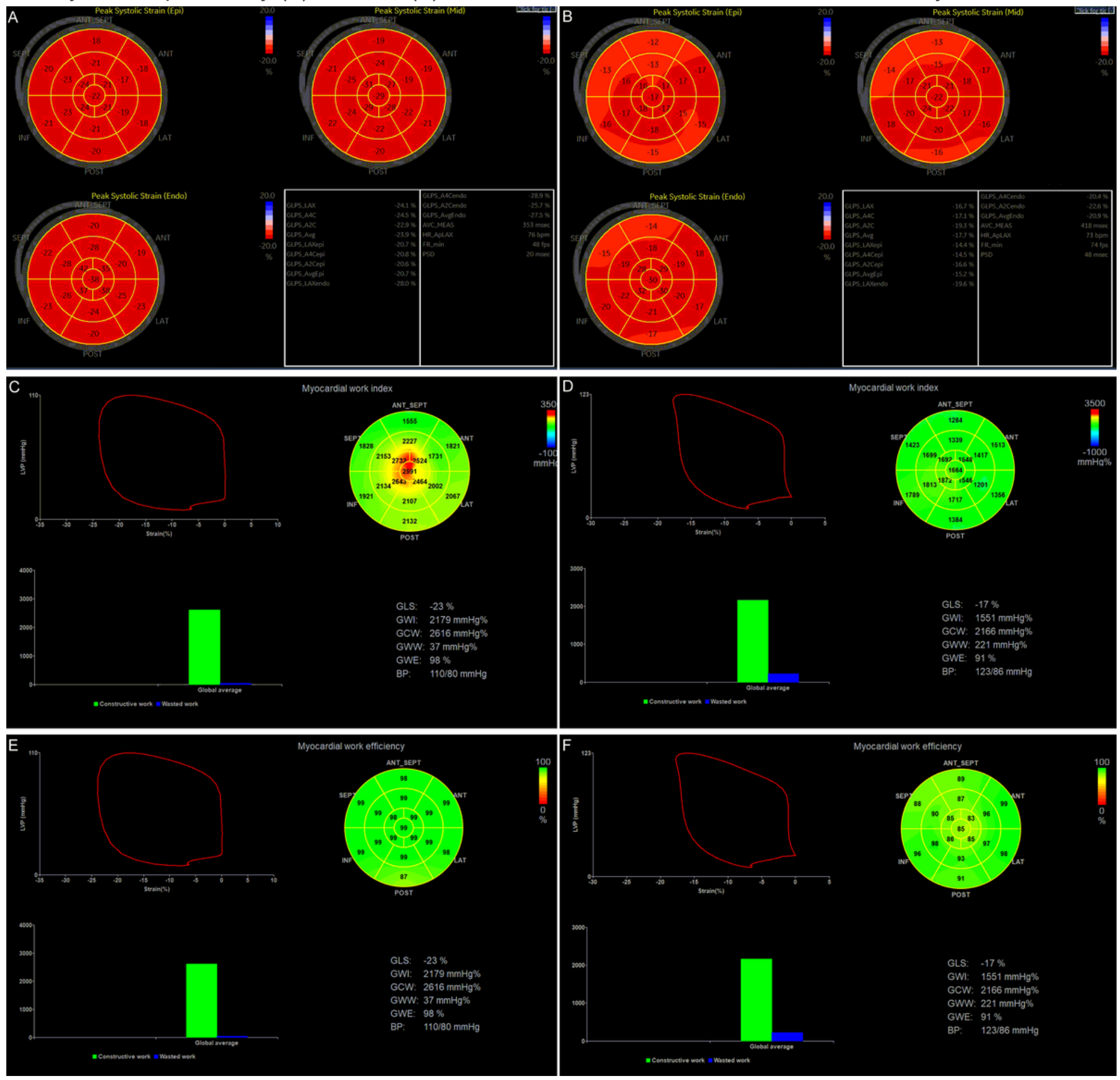

\section{Figure 2}

The bull's eyes of the peak systolic myocardial layer-specific longitudinal strain of normal controls and T2DM patients (A and B). Myocardial work of normal controls and T2DM patients (C, D, E, F). 


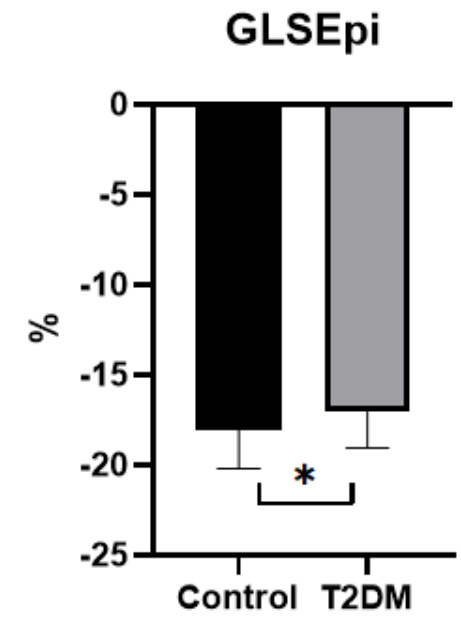

GLSMid

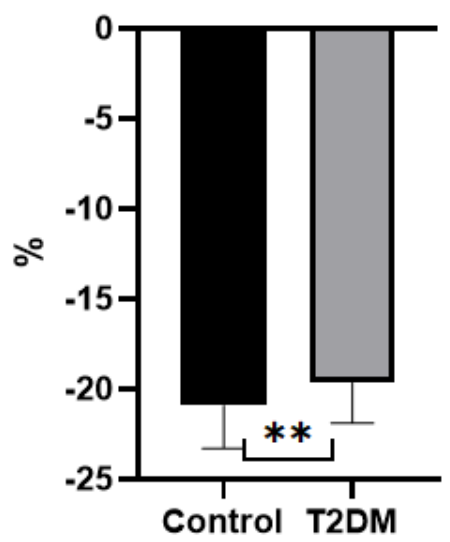

GLSEndo

PSD
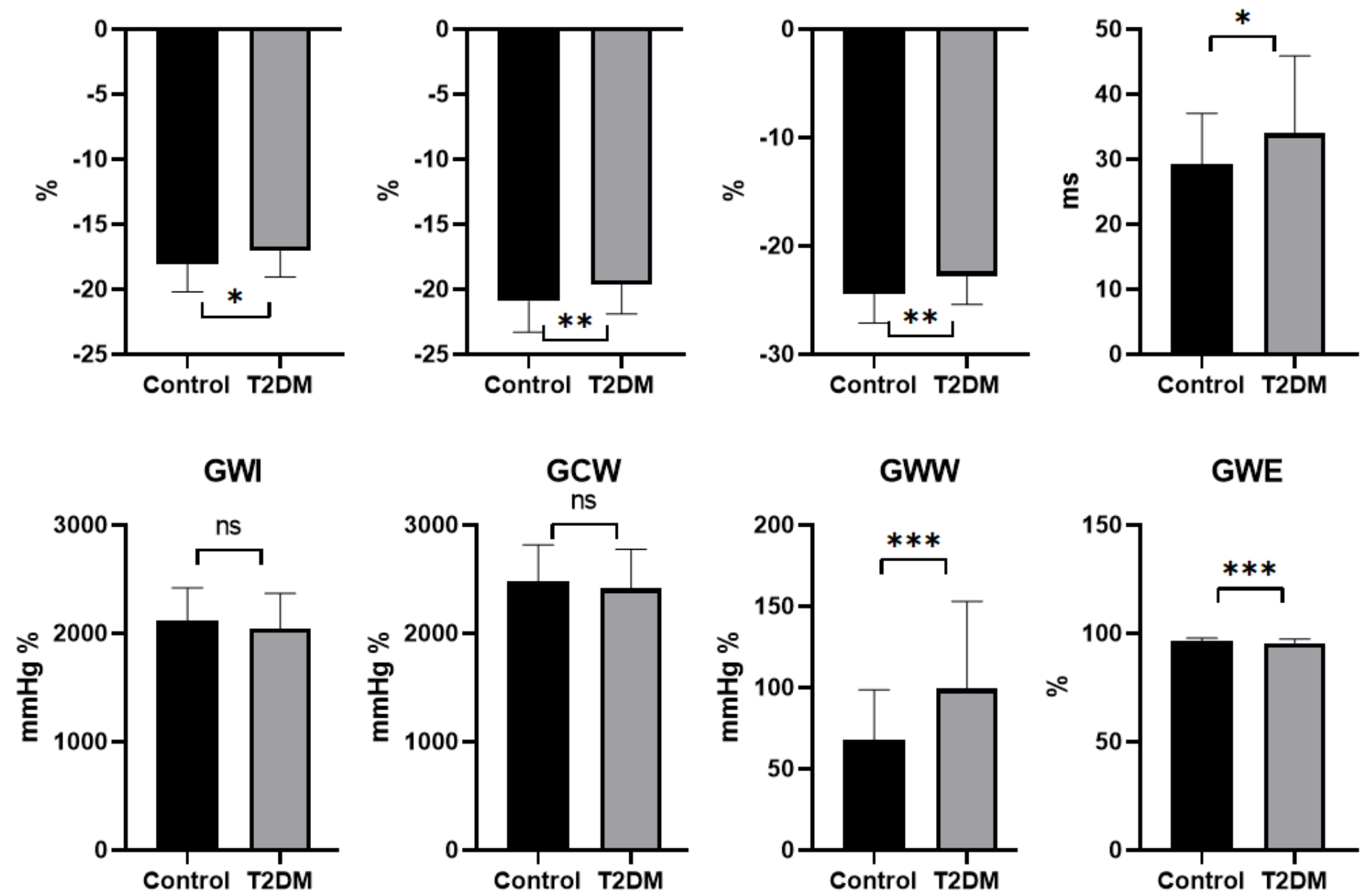

Figure 3

Myocardial layer specific strain, PSD and myocardial work between normal controls and T2DM patients ( ${ }^{\star}$ means $p<0.05$, ${ }^{* *}$ means $p<0.01, * \star *$ means $p<0.001$, ns: non-significant). 
GLSEpi

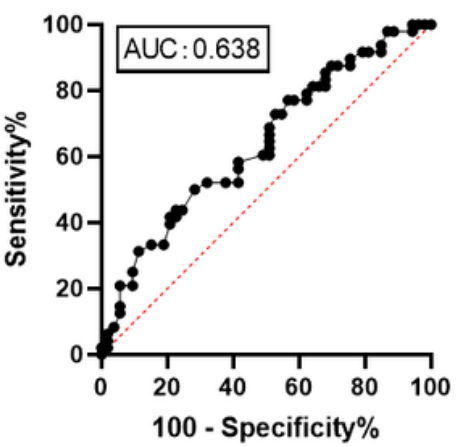

GWI

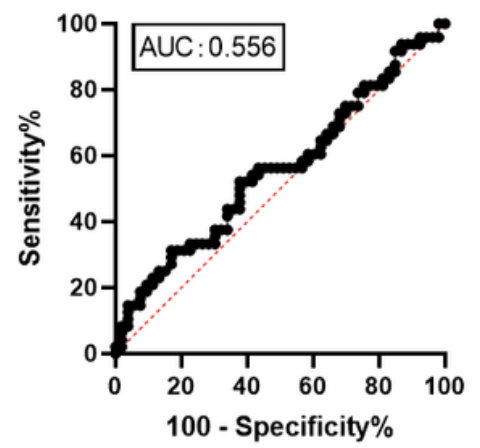

GLSMid

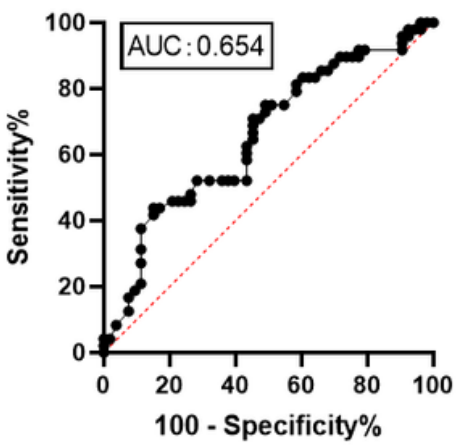

GCW

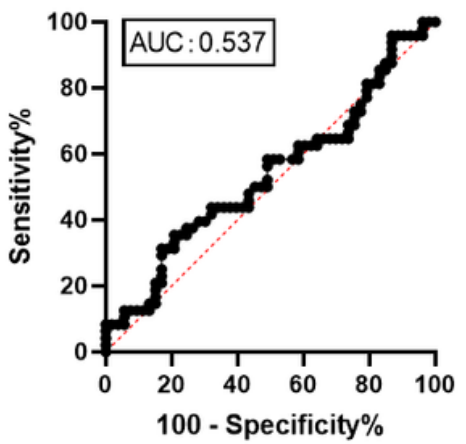

GLSEndo

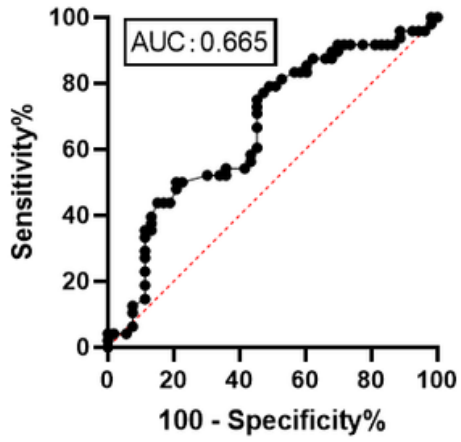

GWW

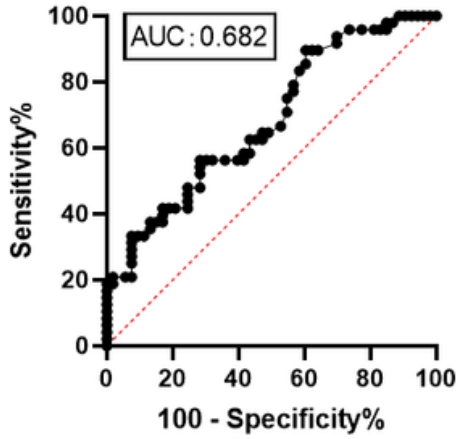

PSD

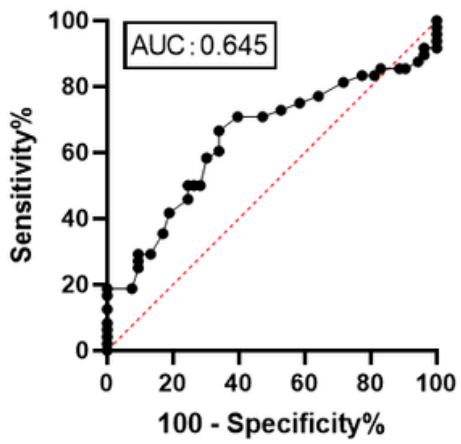

GWE

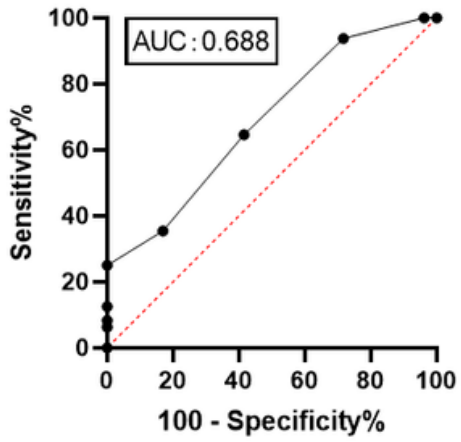

Figure 4

ROC analysis for detecting the accuracy of LV dysfunction in T2DM patients. The area under ROC curve values for GWW and GWE in T2DM patients were a litter higher than myocardial layer specific strain, PSD, GWI and GCW.
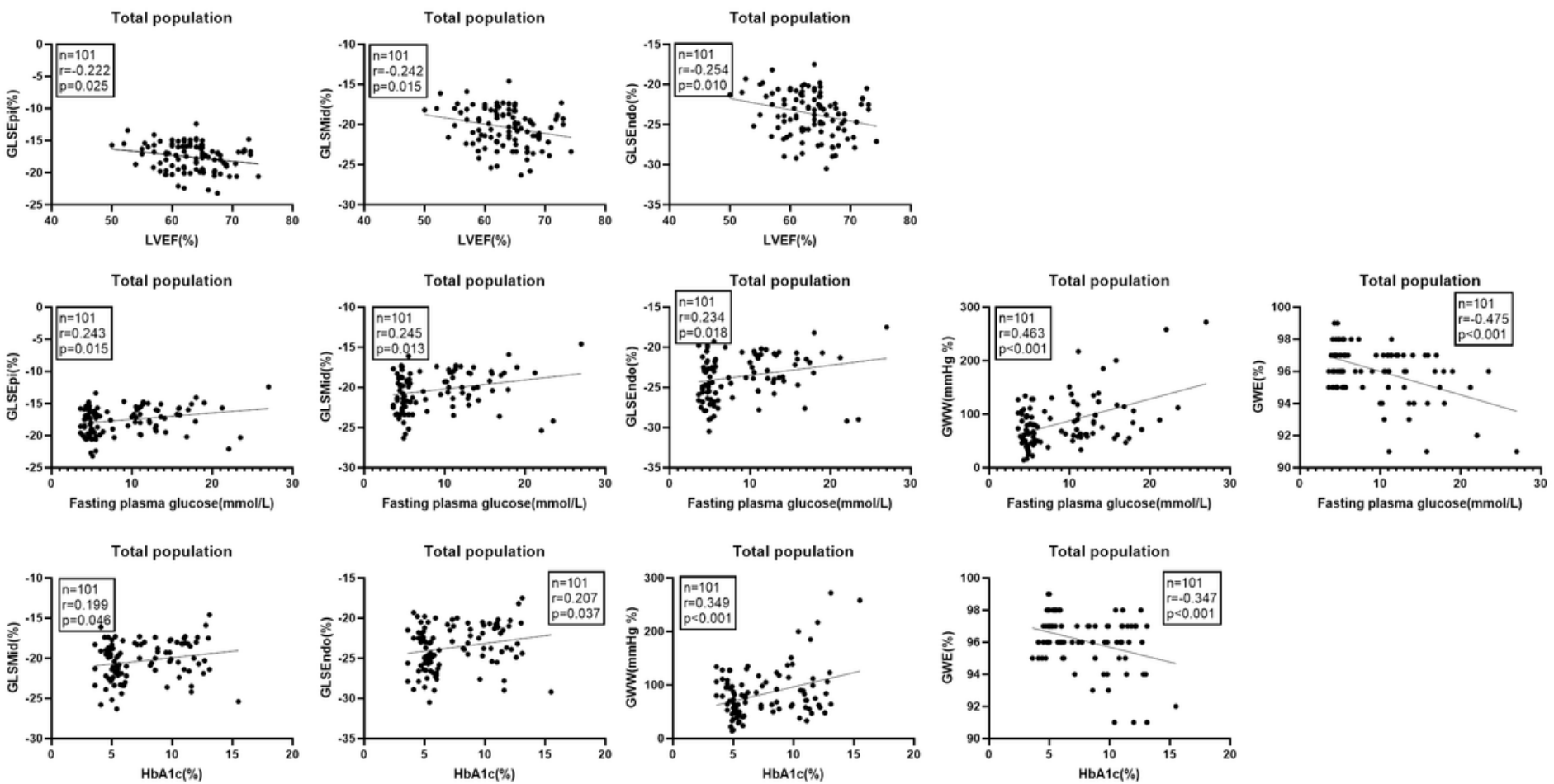
Figure 5

Correlation tests showed in total population, LVEF was negatively correlate with GLSEpi, GLSMid, and GLSEndo. Fasting plasma glucose was positively correlate with GLSEpi, GLSMid, GLSEndo and GWW, while negatively correlate with GWE. HbA1c was positively correlate with GLSMid, GLSEndo and GWW, while negatively correlate with GWE.

Control

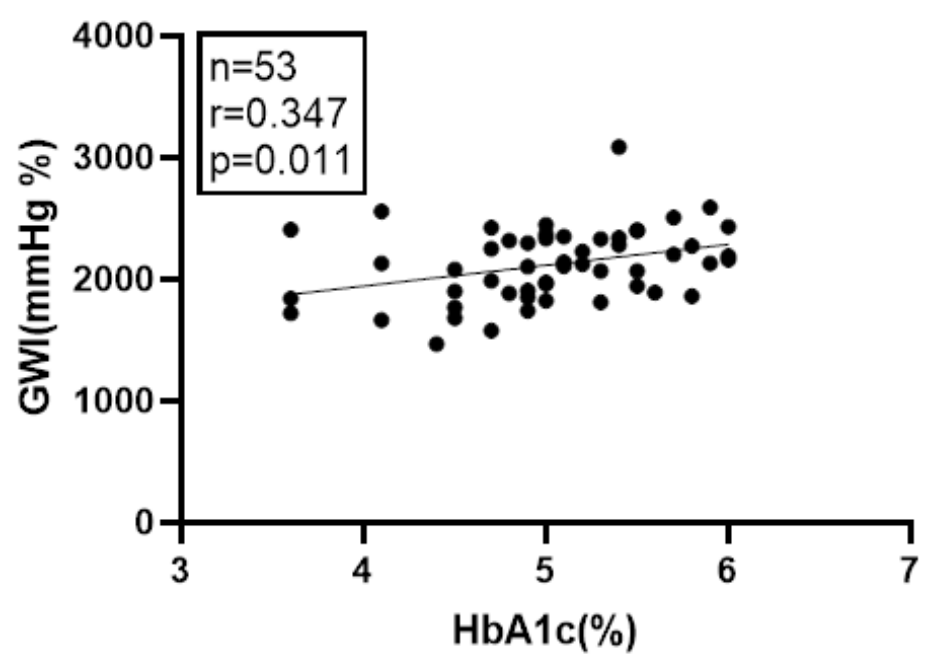

Control

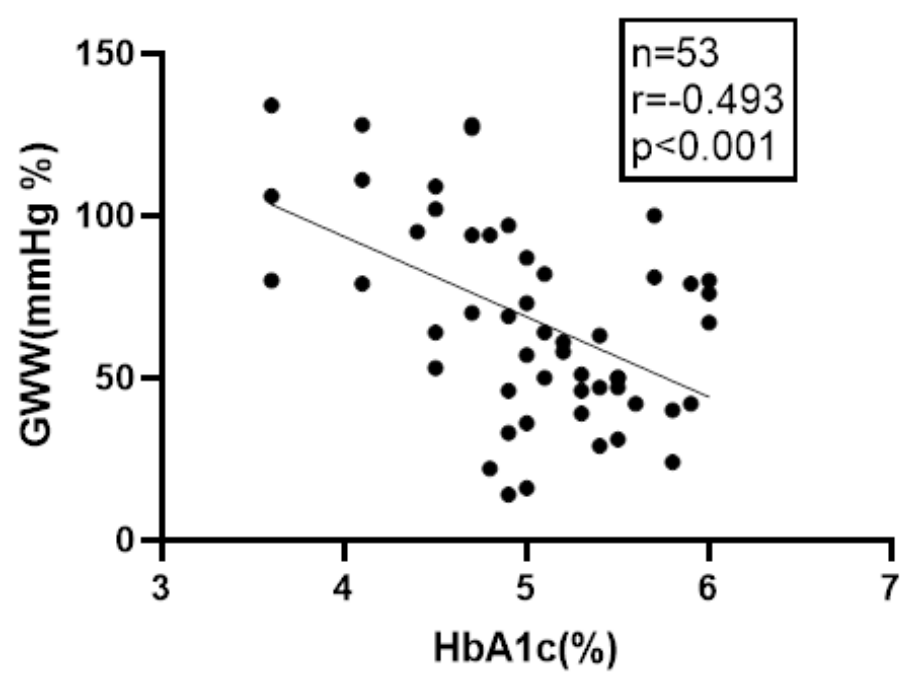

Control

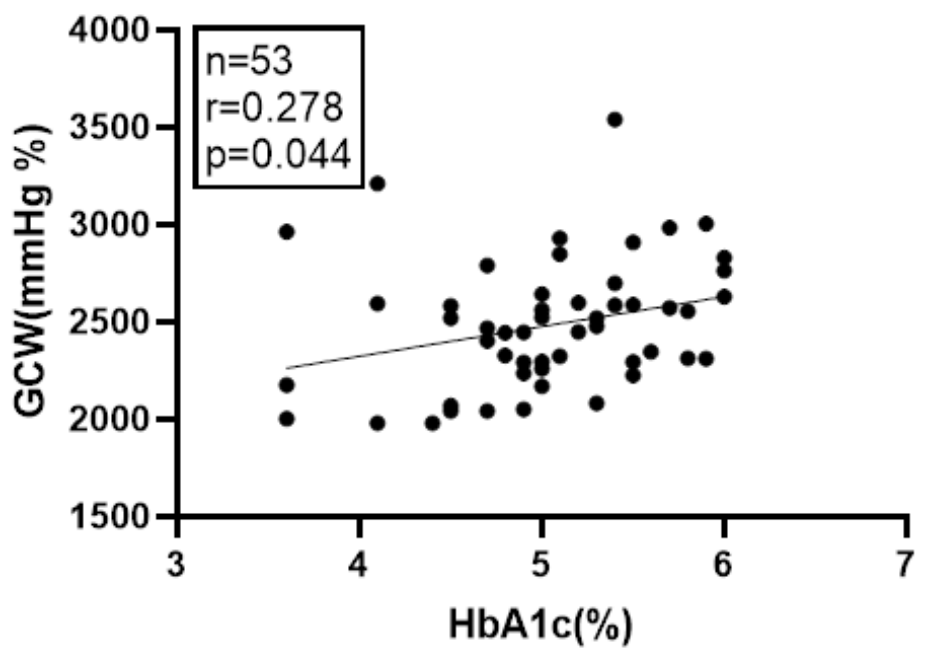

Control

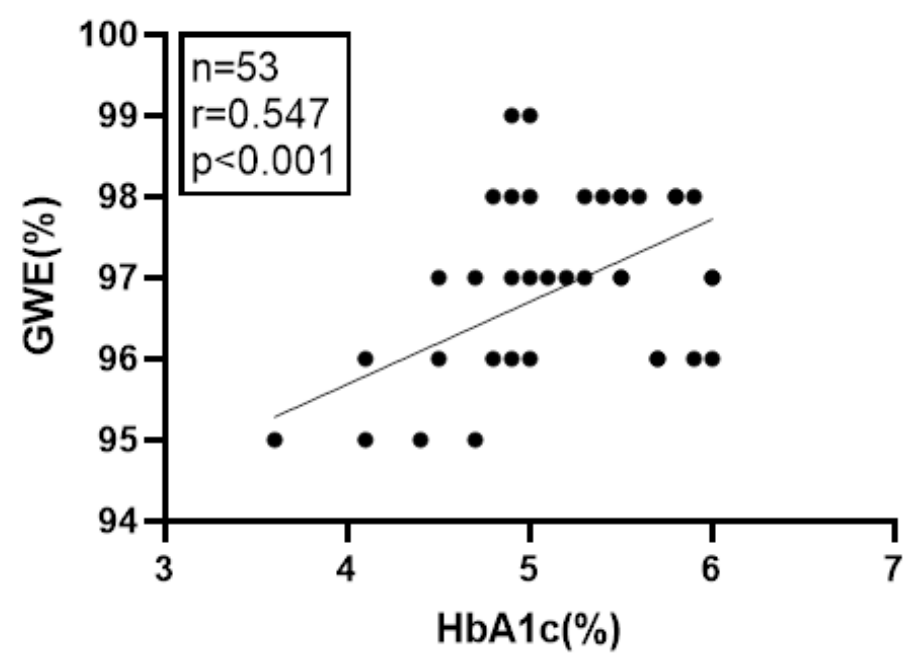

Figure 6

Correlation tests showed in normal controls, $\mathrm{HbA} 1 \mathrm{c}$ was positively correlate with $\mathrm{GWI}$, GCW and GWE, while negatively correlate with GWW. 
T2DM patients

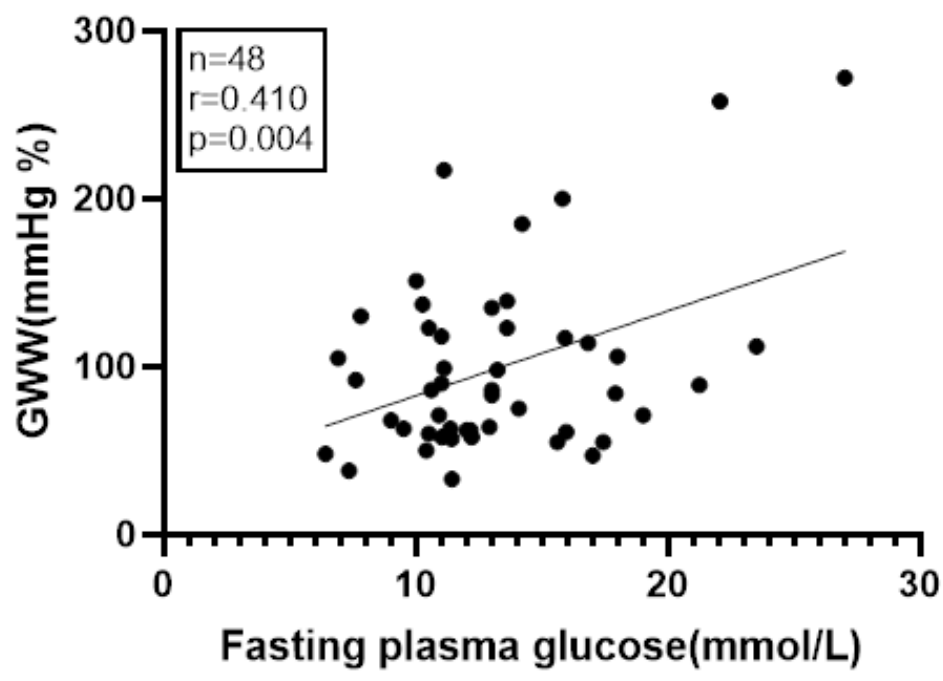

T2DM patients

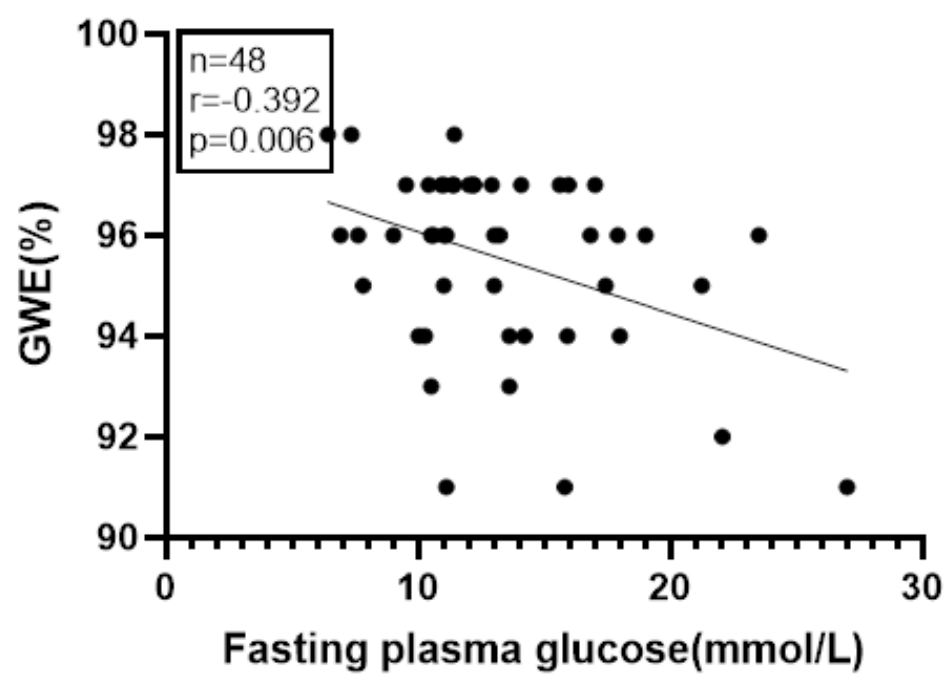

\section{Figure 7}

Correlation tests showed in T2DM patients, fasting plasma glucose was positively correlate with GWW, while negatively correlate with GWE. 